To be published in the Proceedings of the VIIIth International Conference on High

Energy Accelerators, CERN,

Geneva, Switzerland

\begin{abstract}
AN ANALOG METHOD FOR MEASURING THE LONGITUDINAL COUPLING IMPEDANCE OF A RELATIVISTIC PARTICLE BEAM WITH ITS ENVIRONMENT
\end{abstract}

A. Faltens, E. C. Hartwig, D. Möhl, and A. M. Sessler August 2, 1971

AEC Contract No. W-7405-eng-48

TWO-WEEK LOAN COPY

This is a Library Circulating Copy which may be borrowed for two weeks. For a personal retention copy, call Tech. Info. Division, Ext. 5545

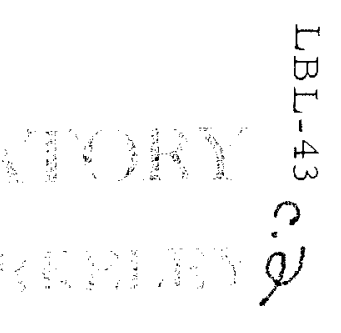




\section{ITS ENVIRONMENT}

A. Faltens, E. C. Hartwig, D. Möhl, ${ }^{+}$and A. M. Sessler

Lawrence Berkeley Laboratory, ${ }^{* *}$ University of California, Berkeley, California 94720

\section{Abstract}

The stability of a coasting beam against selfbunching (negative mass instability) may be expressed in terms of a beam coupling impedance. The impedance has contributions from self-fields, wall impedance, and curvature effects. This paper describes procedures for measuring the wall and curvature contributions to the coupling impedance by means of an analog in which the beam is replaced by a conductor propagating a TEM-like mode. Conditions are derived under which the measurements are valid, various measurement procedures are described, and results of the application of the method to the compressor of an electron ring accelerator are reported.

\section{Introduction}

Stability of a coasting particle beam against self-bunching ("negative mass instability") is necessary for the successful operation of proton storage rings and electron ring accelerators as well as for the efficient performance of many conventional cyclic particle accelerators. The essential element in determining azimuthal beam stability is the beam longitudinal coupling impedance, which is a measure of the coupling of the beam with itself resulting from the interaction of the beam with its environment?).

Although for a variety of structures the coupling impedance can be theoretically computed [see Ref. (2) for numerous examples, as well as an extensive bibliography of the original literature], there are many structures of practical interest which are too complicated to be readily amenable to theoretical analysis. This paper is concerned with a method for measuring the beam longitudinal coupling impedance. The essence of the method is the determination of the propagation velocity of a TEMlike wave in the beam chamber by means of a phase shift measurement. Simulation of the beam by a wire (as we do) has been previously employed in measuring lumped coupling impedances. The techniques employed in these previous measurements may be described as a voltage and current measurement. The phase method to be discussed in the present note is more sensitive by at least a factor of 10 , and makes the measurement of small lumped and distributed impedances possible. Thus we present, in this paper, analysis of' a new problem, as well as novel experimental techniques.

Supported by the U. S. Atomic Energy Commission.

+ Permanent address: CHRN, Geneva 23, Switzerland.

**

Formerly the Lawrence Radiation Laboratory.
In See. II there is firstly presented a discussion of the definition of the coupling impedance and its role in beam stability theory. There then follows a review of previous work in which--under certain circumstances-the coupling impedance is related to wall impedance. Then, in the last part of Sec. II, the properties of a conductor-chamber system are analyzed and it is shown how to deduce the coupling impedance from electrical measurements.

Section III is devoted to a general derivation of the result deduced in Sec. II. The argument in Sec. III is self-contained, more general, and more straightforward than the reasoning employed in Sec. II, but was, of course, preceded by the work described in the earlier section.

Section IV extends the discussion of measurement procedures. In the first part a number of specific techniques are described, and in the second part a description of the application of these techniques to the IJBI electron ring accelerator (ERA) compressor 4 is given 3 .

\section{Impedances}

The three parts of this section are devoted to (1) beam stability theory, (2) the relation between beam and wall impedance, and (3) the transmission line characteristics of a conductor-chamber system.

\section{Beam Stability Theory}

The stability of a coasting particle beam against self-bunching ("negative mass instability") may be expressed in terms of a coupling impedance $\mathrm{z}_{\mathrm{n}}$ defined $\mathrm{by}^{2}$

$$
z_{n}=-\frac{2 \pi R E_{n}}{I_{n}} \text {. }
$$

Here $E$ is the nth harmonic of the azimuthal electric field at the beam, $I_{n}$ is the nth harmonic of the beam current and $R$ the orbit radius.

As a rule of thumb the beam is stable against self-bunching at frequency $n \omega_{\text {rev }}$ if $\left.{ }^{1,}, 4\right)$

$$
\frac{\left|z_{n}\right|}{n} \lesssim \frac{\eta y U_{0}}{I_{0}}\left(\frac{\Delta E}{E}\right)^{2},
$$

where

$$
\omega_{\text {rev }}=\frac{B c}{R}
$$

is the particle revolution frequency,

$$
r_{1}=\left|\frac{1}{\gamma_{t}^{2}}-\frac{1}{\gamma^{2}}\right|,
$$

with $\gamma_{t}$ the transition energy in units of $m_{0} c^{2}$ 
and $\gamma$ the particle energy in units of $m c^{2}$, so that $E=\gamma \mathrm{m}_{0} \mathrm{c}^{2}, \quad U_{0}=\mathrm{m}_{\mathrm{c}^{2}} / \mathrm{e}=0.511 \mathrm{MV}$ for electrons and 938. MV for protons, $I_{0}=\mathrm{Ne} f$ is the circulating beam current, and $\mathrm{E} / \mathrm{E}$ rev the full width of the beam energy distribution at half maximum.

\section{Beam and Wall Impedances}

There are a number of different phenomena which contribute terms to the beam coupling impedance $z_{n}$. There always is a contribution from selffield effects $\left(z_{n S}\right)$. This term is usually computed under the assumption of a perfectly conducting smooth wall surrounding a beam moving in a straight line, and in the long wavelength limit $\left[\lambda_{n}=2 \pi R / n\right.$ $\gg 2 \pi \mathrm{b} / \gamma$, with $\mathrm{b}$ the chamber radius]:

$$
z_{n S}=\mu_{0} c \frac{i n}{\beta \gamma^{2}}\left(\frac{1}{2}+\frac{2 \pi K_{0}}{\mu_{0} c}\right),
$$

where $\mathrm{K}$ is the characteristic impedance of a transmission line consisting of the beam as the central conductor and the perfectly conducting chamber as the outer conductor. For a beam of average radius, $a_{1}$ ) moving inside a chamber of average radius, $b,: 1$ )

$$
\frac{2 \pi K_{0}}{\mu_{0} c}=\ln \frac{b}{a} \text {. }
$$

The nonzero surface impedance of the chamber walls contributes an additional term $\left(\mathrm{z}_{\mathrm{nW}}\right)$ to the coupling impedance $z .5,6)$ In the long wavelength limit it has been shown 7 ) that $z_{n W} / 2 \pi R$ is simply equal to the surface impedance per unit length $Z^{\prime}$. The impedance $Z^{\prime}$ is the same quantity that appears in the usual transmission line theory, while $2 \pi \mathrm{bZ}$ ' is the wall impedance of traveling wave tube theory $\left[-\mathrm{E}_{\mathrm{z}}(\mathrm{b}) / \mathrm{H}_{\varnothing}(\mathrm{b})\right.$ in a straight tube of radius $b$ ].

A lumped wall impedance, $z$, due, for example, to a narrow cavity or a discontinuity in chamber cross section will, in the long wavelength limit, contribute to $z_{n}$ a term, $z_{n L}$, just equal to $z$.

A final contribution, $Z_{n C}$, to the coupling impedance $z_{n}$ arises from the curvature of the beam major radius. For a chamber with full height, $H$, much less than-its full (radial) width, and for mode numbers, $n_{8}$ such that $n \ll \pi R / \beta H$ it has

$$
z_{n C}=\frac{i \mu_{0} \text { e } \beta}{2}\left(\frac{H}{\pi R}\right)^{2} \text {. }
$$

Generally, $z_{n C}$ is a rather difficult quantity to evaluate analytically. In conventional synchrotrons $Z_{n C} i s$, of course, a negligible term, but in electron ring accelerators it can be the dominant term in $\mathrm{z}_{\mathrm{n}}$.

In summary, in the long wavelength limit

$$
z_{n}=z_{n S}+z_{n W}+z_{n J}+z_{n C} \text {, }
$$

with $z_{n S}$ a term of order $\gamma^{-2}$ and hence quite small in the relativistic limit. ${ }^{*}$ The remaining terms we will show to be measurable by an analog method.

3. Transmission Line Characteristics of a Conductor Chamber System

Consider the transmission line formed by inserting a conductor into the vacuum chamber at the beam position. For the remainder of this section we neglect curvature terms, and hence we consider a straight transmission line and may employ conventional cireuit theory 9 ).

First, consider a smooth chamber without lumped impedances. Let a unit element of the line be represented by the equivalent circuit shown in Fig. 1 .

Fig. 1. Unit element of the conductor-chamber system. The inductance per unit length of the ideal transmission line, $L^{\prime}+L^{\prime}$, has -arbitrarily--been split into two parts. I The capacitance per unit length of the ideal line is $C^{\prime}$. The impedance per unit length of the outer wall is $Z^{\prime}$, and $Z_{2}^{\prime}$ is the impedance per unit length of the central conductor.

Let

$$
\begin{aligned}
& L_{t}^{\prime}=L^{\prime}+L_{2}^{\prime}, \\
& Z_{t}^{\prime}=Z^{\prime}+Z_{2}^{\prime} .
\end{aligned}
$$

Here the index 2 refers to the central conductor, and quantities without index refer to the properties of the outer wall. The inductance per unit length of the ideal transmission line is designated by $L^{\prime}$ and has been--arbitrarily--split into the two terms $L^{\prime}$ and $L^{\prime}$. The term $Z^{\prime}$ is due to the finite surface impedance of the central conductor. The impedance per unit length of the outer wall is $Z^{\prime}$, and this is the quantity that we wish to measure. The familiar equations for the TEM current $I$ and potential U are

We assume here and in the following that for the unstable wave $\beta_{\mathrm{W}}=\omega / \mathrm{kc}=\beta$, cf. Ref. (5).

**

In agreement with engineering textbooks we write in the present subsection the time dependence as $e^{+j \omega t}$ instead of the "theorist's convention" $e^{-i \text { ict }}$. Results can be converted by replacing $j$ with $-i$. 


$$
\begin{aligned}
& \frac{d U}{d X}=-\left(j w L_{t}^{\prime}+Z_{t}^{\prime}\right) I, \\
& \frac{d I}{d X}=-j w C^{\prime} U,
\end{aligned}
$$

which are valid to first order in $Z_{t}^{1} / j u L_{t}^{\prime}$.

The main parameters characterizing the line of Fig. 1 are the propagation constant

$$
j \mathrm{k}_{\mathrm{O}}=\left[j \omega C^{\prime}\left(j \omega \mathrm{L}_{t}^{\prime}+z_{t}^{\prime}\right)\right]^{\frac{1}{2}} \approx j \omega\left(\mathrm{L}_{t}^{\prime} \mathrm{C}^{\prime}\right)^{\frac{1}{2}}\left(1+\frac{1}{2} \frac{Z_{t}^{\prime}}{j \omega L_{t}^{\prime}}\right),
$$

and the characteristic impedance

$$
z_{0}=\left(\frac{j \omega L_{t}^{\prime}+z_{t}^{\prime}}{j \omega c^{\prime}}\right)^{\frac{1}{2}} \approx\left(\frac{L_{t}^{\prime}}{c^{1}}\right)^{\frac{1}{2}}\left(1+\frac{1}{2} \frac{z_{t}^{\prime}}{j \omega L_{t}^{\prime}}\right)^{\frac{1}{2}} \text {. }
$$

The transmission line parameters $\mathrm{L}_{1}^{1}, \mathrm{Z}_{\mathrm{r}}^{\prime}$, and $C^{\prime}$ can in principle be calculated or determined by a model measurement. A measurement of $k$ and/or $Z_{0}$ then serves to determine the wall impedance $Z^{\prime}$.

Consider, now, a chamber having a lumped impedance $z$ located at the position $x$. The line equations $(2.8)$ are augmented to become:

$$
\begin{aligned}
& \frac{d U}{d x}=-\left[j \omega I_{t}^{\prime}+z_{t}^{\prime}+z \delta\left(x-x_{0}\right)\right] I, \\
& \frac{d I}{d x}=-j \omega C^{\prime} U .
\end{aligned}
$$

An approximate traveling wave solution of (2.11) is:

$$
\begin{aligned}
& \mathrm{U} \approx \mathrm{U}_{\mathrm{O}} e^{-j \phi(x)}, \\
& I \approx \frac{U_{0}}{z_{0}} e^{-j \phi(x)},
\end{aligned}
$$

with

$\phi(x)=\omega\left(L_{t}^{\prime} C^{\prime}\right)^{\frac{1}{2}} \int^{x}\left\langle 1+\left.\frac{1}{2} \frac{\left[z_{t}^{\prime}+z \delta\left(x-x_{0}\right)\right]}{j \omega L_{t}^{\prime}}\right|_{(2.13)} ^{d x}\right.$.

From (2.13), the phase difference between $\mathrm{x}=0$ and $\mathrm{x}=\mathrm{d}$ is

$\phi(d)-\phi(0) \approx \omega\left(L_{t}^{2} C^{\prime}\right)^{\frac{i}{2}} d\left(1+\frac{1}{2} \frac{Z_{t}^{\prime}+Z / d}{j \omega L_{t}^{\prime}}\right)$.

Thus a measurement of phase difference yields information about $\left(z_{t}^{\prime}+z / d\right)$ which is exactly the quantity entering the beam coupling impedance $z_{n}$ (provided $Z^{\prime} \gg Z_{2}^{\prime}$ ).

Finally, as suggested in Ref. 8, curvature terms appear to enter both the beam problem and the transmission line in the same manner so that a curved line would be expected to have $2 \pi R Z_{t}^{\prime}$ augmented by the term $Z_{\text {. Th }}$. This hypothesis will be shown, in the next section, to be correct.

\section{Formal Derivation}

In this section we establish the relation between a beam coupling impedance and the phase velocity of a corresponiding TEM-Iike mode on an analog transmission line. The method of proof is an extension of a technique developed by $V$. K. Neil ${ }^{10}$. It is not as straightforward as a Green's function analysis, but provides more physical insight into the problem while yielding the same result.

We consider--separately--the two situations of a beam in a conducting chamber and a wire in the same chamber. Nonzero chamber wall impedances and curvature of the beam (and wire) are included. The essence of the method is to replace the wire by suitably chosen fictitious currents which are then found to be closely similar to the beam sources, and hence allow the deduction of a relation between the two situations.

We start from the wave equation for the sealar and vector potential which in the Lorentz gauge and for a time dependence $e^{-j \omega t}$ we write as

$$
\begin{aligned}
& \nabla^{2} A+(\omega / c)^{2} \underset{\sim}{A}=-\mu_{0} \underset{\sim}{j} \\
& \nabla^{2} \Phi+(\omega / c)^{2} \Phi=-\left(1 / \epsilon_{0}\right) \rho .
\end{aligned}
$$

The vector potential $\underset{\sim}{A}$ and the scalar potential $\Phi$ are interrelated by the Lorentz condition

$$
\Phi=\left(c^{2} / i \omega\right) \underset{\sim}{\nabla} \underset{\sim}{A},
$$

so it is sufficient to solve the wave equation for A.

Using cylindrical coordinates with the $\theta$ direction as the direction of particle motion we write the azimuthal electric field at the beam (or wire) surface as

$$
\begin{aligned}
& E_{\theta}=i \omega A_{\theta}+\frac{i c^{2}}{\omega R} \frac{\partial}{\partial \theta}(\underset{\sim}{\sim} \underset{\sim}{A}) \\
& =i \omega\left(1+\frac{c^{2}}{\omega^{2} R^{2}} \frac{\partial^{2}}{\partial \theta^{2}}\right) A_{\theta}+E_{1}
\end{aligned}
$$

$E_{1}=i \frac{c^{2}}{\omega R} \frac{\partial}{\partial \theta}\left(\left.\frac{1}{R} \frac{\partial}{\partial r}\left(r A_{r}\right)\right|_{r=R}+\left.\frac{\partial A_{z}}{\partial z}\right|_{z=0}\right)$.

In a straight, perfectly conducting structure, $A_{r}=A_{z}=0$, and hence $E_{1}=0$. Curvature and non$z$ erro wăll impedance will contribute to $\mathrm{E}_{\theta}$ through $E_{1}$ as well as through a changed $A_{e}$. Provided, however, the sources vary as $e^{i n \theta}$ and the chamber is smooth as a function of $\theta$ (no lumped impedances), the potentials will also vary as $e^{i n \theta}$ and the $A_{\theta}$ term in $E_{\theta}$ contributes a term proportional to 


$$
\left.\left(1-\frac{c^{2} n^{2}}{\omega^{2} R^{2}}\right)=\left(1-\frac{1}{\beta^{2}}\right)=-\frac{1}{\beta^{2} \gamma^{2}}\right),
$$

and hence negligibly in the relativistic limit.

Consider, firstly, the beam as a source. Neglecting the finite size of the beam, we may write

$$
\begin{aligned}
j=j_{\theta} & =I_{n} \delta(r-R) \delta(z) e^{i(n \theta-a t)}, \\
\rho & =j_{\theta} \frac{n}{\omega R},
\end{aligned}
$$

with $w \approx n \beta c / R$. Solution of (3.1) with the source (3.4) and appropriate boundary conditions on the chamber walls would yield, via (3.3), the coupling impedance $Z_{n}$, as defined by (2.1).

Consider, now, the situation in which the chamber has a perfectly conducting wire replacing the beam. The field boundary conditions on the wire are that $\mathrm{E}_{\|}=0$ and

$$
\begin{aligned}
& j_{\text {surf }}=H_{11}, \\
& \rho_{\text {surf }}=\epsilon E_{\perp},
\end{aligned}
$$

where $\rho_{\text {surf }}$ and $j_{\text {surf }}$ are the surface charge and current on the wire. We may regard this charge and current as a source for the potential $A$, which is then determined by (3.1) along with the boundary conditions on the chamber. A proper solution has the fields obtained from $\underset{\sim}{A}$ consistent with the assumed sources.

We obtain the conducting wire solution by an iterative procedure in which zero-order sources produce first-order fields which are required to satisfy $E_{\theta}=0$. Using an ideal TFM field as a guide we take zero-order sources as:

$$
\begin{aligned}
& \underset{\sim}{j}=j_{\theta}=I_{n} \delta(r-R) \delta(z) e^{i(\tilde{n} \theta-\omega t)}, \\
& \rho=j_{\theta} \frac{\tilde{n}}{R \omega} .
\end{aligned}
$$

It will be noted that the "azimuthal wave number" $\tilde{\mathrm{n}}$ is related to the propagation constant $(2.9)$ of Sec. II. 3 by $k=\tilde{n} / R$. Solving (3.1), with the proper chamber boundary conditions yields a potential A and then, from (3.3),

$$
E_{\theta}=i \omega\left(1-\frac{c^{2} \tilde{n}^{2}}{\omega^{2} R^{2}}\right) A_{\theta}+E_{1}(\tilde{n}) \text {. }
$$

Demanding that $E_{\theta}=0$ yields an equation for $\tilde{n}$.

$$
\text { For } \underset{\sim}{\sim} \text { perfectly conducting smooth straight }
$$
chamber $\tilde{\mathrm{n}}=\widetilde{\mathrm{n}}_{\mathrm{O}}$, with

$$
\tilde{n}_{0}=\frac{\omega R}{c} \text {, }
$$
and $\mathrm{A}_{\text {arfas only the component }} A_{\theta}=A_{\theta O}$, which at
the surface conductor is

$$
A_{\theta O}=L_{t}^{\prime} I_{n} e^{i\left(\tilde{n}_{0} \theta-t\right)} .
$$

Here $I_{t}^{\prime}$ is the inductance per unit length of the ideal transmission line. From (3.6) we obtain the first order shift in $\tilde{\mathrm{n}}$ :

$$
\frac{\Delta \tilde{n}}{\tilde{n}}=\frac{1}{2} \frac{E_{1}\left(\tilde{n}_{0}\right)}{i \omega I_{t} I_{n}} \text {, }
$$

where $\Delta \tilde{\mathrm{n}} \equiv \tilde{\mathrm{n}}-\tilde{\mathrm{n}}_{0}$.

The quantity $E_{1}(\tilde{n})$ is approximately equal to $\mathrm{E}_{(\mathrm{n}}(\mathrm{n})$ [as determined by the solution of (3.1) with the bearn sources (3.4)] since the assumed sources (3.5) are almost the same as the sources (3.4). In fact, provided that

and

$$
1-\beta \approx \frac{1}{2 \gamma^{2}} \ll 1 \text {, }
$$

$$
\frac{\Delta \tilde{n}}{\tilde{n}} \ll 1,
$$

the difference in the azimuthal wave number of the two sources is negligible and $\mathrm{E}_{\mathrm{l}}\left(\widetilde{\mathrm{n}}_{0}\right)$ may be replaced by $E_{7}(n)$. This replacement would be inaccurate if the chamber had very sharp resonances, so that the response at wavelengths $(2 \pi \mathrm{c} / \omega) B$ and $(2 \pi c / \omega)(1+\tilde{n} / \tilde{n})^{-1}$ were very different. When (3.10) is satisfied, and employing the definition (2.1), we have from (3.9):

$$
z_{n W}+z_{n C} \approx-4 \pi \operatorname{Ri\omega } L_{t}^{\prime}\left(\triangle \tilde{n} / \tilde{n}_{0}\right) .
$$

Equation (3.11) will be recognized as a generalization of (2.9) through the inclusion of $z_{n C}$.

The self-field term, $z_{\text {, }}$, can in general be estimated with sufficient accuracy, or it might be determined through an auxiliary measyrement. However, since $Z$ exhibits $a \mathrm{I} / \mathrm{\gamma}^{2}$ cancellation it is negligible $\mathrm{n}$ in the relativistic limit. Hence a measurement of $\Delta \widetilde{n} / \widetilde{n}$ is a direct measure of the coupling impedance terms which are the dominant terms at relativistic energy. Furthermore, these terms are the most difficult terms to estimate theoretically.

Extension of the derivation to include lumped impedances follows arguments employed in Sec. II. 3 and will not be detailed here.

\section{Measurements}

In this section we first describe a number of experimental techniques which may be employed to measure the longitudinal coupling impedance, and then describe the application of the techniques to the LBL-ERA compressor 4.

At first sight, the analog transmission line might be used to yield a coupling impedance via a direct measurement of the longitudinal electric field $E_{\theta}$. However, the large transverse fields 
preclude such a measurement. Consequently, more refined methods must be employed, and wo describe three possibilities in the first part of this section.

In general it is convenient to employ a highly conducting analog wire $\left(Z^{\prime} \ll Z^{\prime}\right)$, but if the propagation constant is strongly frequency dependent it is possible--in principle--to choose $Z_{?}^{\prime}$ so as to match both the wavelength and frequency of the analog line to that of the beam. In the experimental work reported in this paper the structure was not resonant in the range of interest, and the matching of propagation constants was not required.

\section{Three Measurement Techniques}

Measurement of the properties of a transmission line is a highly developed subject and need not be detailed here. We confine ourselves to brief descriptions of three techniques.

A. Pulse response. If a short pulse is transmitted along the conductor-chamber system, reflections will occur from discontinuities (lumped wall impedances). In addition the transmitted pulse is distorted due to the presence of both lumped and distributed wall impedances which make the line dispersive.

The measurement of the pulse response is most useful for locating the position of discontinuities by timing of the reflected pulse and also for revealing the presence of strongly resonant coupling impedances. It is a relatively simple technique and is useful for preliminary exploration of a chamber, and for comparative studies of chambers. Unwanted lumped impedances, in the ERA compressor 4, were eliminated using this technique.

B. Standing wave measurements. Neglecting lumped wall impedances, the input impedance of a line of length $\ell$ is given by

$$
z_{i}=z_{0} \frac{\cos k_{0}^{l}+j r \sin k_{0}^{l}}{r \cos k_{0}^{l}+j \sin k_{0}^{l}}
$$

where $r=z_{0} / z_{I}, z_{L}$ being the terminating load impedance.

Either for a shorted $\left(z_{1}=0\right)$ or an open $\left(z_{T}+\infty\right)$ line, the input impedance exhibits sharp resonances. The $Q$ value of these resonances may be used to determine the resistive part of the coupling impedance $z_{n}$, while the shift $(\Delta \omega)$ of the resonant frequency determines the reactive part of $Z_{n}$. (In practice comparative measurements, employing highly conducting chambers, are useful to determine the small shift of resonance.)

These relations may readily be extended to include the influence of a lumped impedance $Z$ at a distance $\ell$ from the feed point. It may be noted that the corresponding expressions for $1 / Q$ and $\Delta \omega$ are the same as in the case of a distributed impedance $z$ 'efe given by:

$$
z_{\text {eff }}^{\prime}=z \frac{\sin ^{2} k_{0}{ }^{i} 1}{i / 2} .
$$

The quantity $\ell$ may be varied by shifting the feed and load point, and hence direct experimental measurement of the effective rall impedance $\left\langle(]_{\text {eff }}^{\prime}\right\rangle=Z / \ell$ is possible.

C. Traveling wave measurements. Neglecting lumped wall impedances, if the conductor chamber system is terminated as closely as possible with its characteristic impedance, then a measurement of the phase difference between two points allows the evaluation of the real part of $k_{p}$ and hence, from (3.12), evaluation of $\operatorname{Im}\left(z_{n C}+z_{n W}\right)$. The simplest measuring technique is to determine the frequencies for which the phase difference is $m \pi$, as under these conditions the influence of reflections (arising from the necessarily imperfect termination) is minimal. It is important that the TEM wave impedance of the beam chamber between the two points of phase measurements be uniform except for short lumps of known position. Phase comparisons between the beam chamber and simple geometry walls for which the propagation velocity or wall impedance is known will then eliminate the need for precise absolute measurements. Phase shifts of $0.1^{\circ}$ are easily detected.

The technique can also be employed to determine the influence of a small lumped impedance in the chamber wall, as follows from (2.14).

\section{Measurements on the ERA Compressor 4}

There was considerable interest in measuring beam coupling impedance in the FRA compressor 4 because it appeared that a negative mass type of instability was severely limiting the current that could be captured into rings. In fact, it was this problem which precipitated the work reported in the present paper. For the compressor 4 design parameters the tolerable impedance at $100 \mathrm{~A}$ circulating current was $\left|z_{n}\right| / n \lesssim 40 \Omega$. The self-field term was $\left|z_{n S}\right| / n \approx 38$.

Chamber walls--actually side walls--which were highly conducting would have been acceptable except for the fact that they would not allow the penetration of the pulsed inflector field (rise time $\approx 20 \mathrm{nsec}$ ) required for injection. Therefore, two general types of walls were developed to satisfy the conflicting requirements of (1) penetration of the inflector pulse, and (2) adequately small coupling impedance for modes $n \geqslant 1$. [Note that the frequency of mode $\mathrm{n}=1$ is $\approx 250 \mathrm{MHz}$.]

The first kind of wall has been a thin metal film--typically a few hundred Angstroms--on an insulating support structure, which gives a resistance of from $I \Omega$ to $50 \Omega$ per square. The usefla resistance range now appears to be $1 \Omega-4 \Omega$ per square, in which range the films are about 0.01 skin depths thick at $250 \mathrm{MHz}$ yet still stop most of the radiation through the walls and provide a low impedance to the beam. The second kind of wall has been made of eircumferential metal hoops, thick 
compared to a skin depth, which are broken at one point and capacitively bridged so as to make a wall which is conductive at high frequencies and nonconductive at low frequencies. The resistive metal film walls have the disadvantage that they have a real part of the impedance $z_{n}$ equal to about 6 times the ohms per square value in the interesting resistance range of a few ohms per square; the hooped walls have the disadvantage that they have resonances. Both types of walls are sufficiently complicated to make an experimental measurement of their properties desirable.

Because of the requirement that $\left|z_{n}\right| / n \lesssim 40 \Omega$, we have had to measure $\left|z_{n}\right|$ with an accuracy of a few ohms, but this was easily possible by means of the methods discussed above.

The characteristic impedance of the equivalent line was close to $50 \delta$ and has been matched to this value by slightly increasing the diameter of the central conductor beyond the minor beam diameter. In most measurements the conductor covered almost the full $360^{\circ}$ of the compressor. Measurements were taken in the range of $50 \mathrm{MHz}$ to $1000 \mathrm{MHz}$. This range was chosen because beam observations had indicated that the instability was dominated by low modes.

The measurements performed were either pulse or traveling wave measurements. In most cases the walls under consideration were compared to a solid aluminium wall of very low surface resistance.

Pulse measurements on a $50 \Omega$ per square wall revealed a considerable difference from the behavior observed with a highly conducting wall. This difference was attributed to insufficient shielding (i.e., a large $z_{n C}$ ). To confirm this assumption a traveling wave was sent through the conductor and the radiation outside the compressor was detected. It was found that the transparency of the wall was sufficiently large to explain the effect. The radiation impedance of an unshielded beam by itself is several times greater than the 40 \& limit, so that good shielding is a prerequisite of the walls.

Pulse type measurements with a $1 \delta$ per square wall, a $2 \&$ per square wall, and a hooped wall showed little difference from a solid metal wall. Figure 2 gives an example of the reflections which occur in the presence of a solid and a hooped metal wall. It is noted that most of the reflections are common to the two walls. They result mainly from junction discontinuities at the two ends, where the conductor is connected to 50 s cables.

Whereas the pulse measurement is relatively coarse and can easily only detect impedance differences greater than $l \Omega$, phase shift measurement can detect differences of $0.1^{\circ}$ per circumference corresponding to a change of $0.2 \delta$ in the coupling impedance. Figure 3 gives an example of such a measurement. It will be noted that at the frequencies of interest ( $250 \mathrm{MHz}-500 \mathrm{MHz}$ ) the differences between a hooped and a solid metal wall, and between a $2 \Omega$ per square film and a solid metal wall are less than 2 degrees per circumference [corresponding

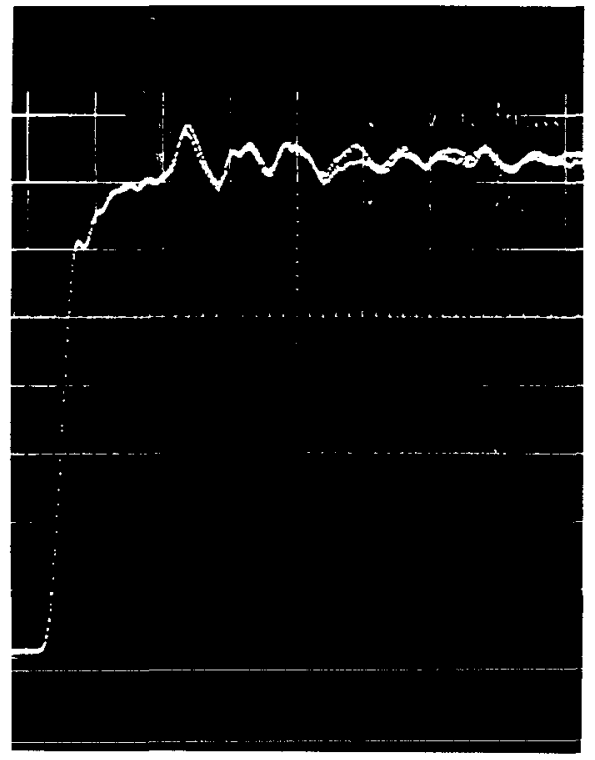

Fig. 2. Reflection of a steep pulse from the simulating conductor in the ERA compressor-4 chamber. Low impedance solid metal wall (lower trace) and 28 per square hooped sidewall. Time scale: 2ns/div.

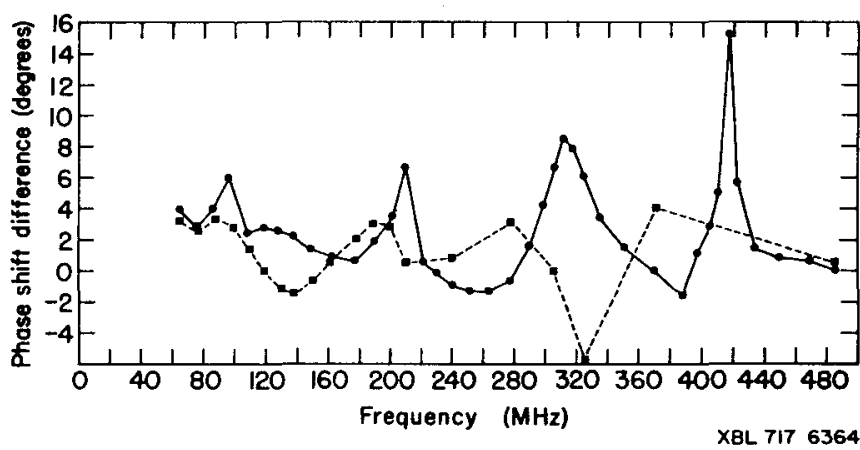

Fig. 3. Measured values of the phase shift difference between hooped walls and solid aluminium walls (-), and between $2 \delta$ per square walls and solid aluminium walls (-.). The conductor simulating the beam covered (almost) the full circumference of the ERA compressor. The phase was detected at the two ends of the conductor.

to about 4 s in the coupling impedance]. No great care was taken to reduce the reflections due to mismatch at the two ends of the conductor. The peaks in the two curves reflect the fact that the phase shift is most sensitive to reflections at frequencies for which the phase difference is an odd multiple of $\pi / 2$ and most insensitive if this difference is $m \pi$. The measurements at $250 \mathrm{MHz}$ and 
$500 \mathrm{MHz}$ are quite reliable, because there $\Delta \phi \simeq 2 \pi$ and $4 \pi$.

The measurements are in an early stage, and the techniques are still being improved. From the limited data obtained so far we have gained confidence that the measuring methods are reliable. Furthermore, by means of repeated design and measurement, we have arrived at the sidewalls described above, which we believe to be suitable for the containment of intense electron rings. Beam experiments, employing these wall, are scheduled for the following months.

\section{References}

1. C. E. Nielsen, A. M. Sessler, and K. R. Symon, International Conference on High-Energy Accelerators, CERN, 1959, p. 239; and A. A. Kolomenskij and A. N. Lebedev, ibid, p. 115.

2. A. M. Sessler, Proceedings of the 1971 Particle Accelerator Conference, IEEE Trans. Nucl. Sci. NS-18 \#3, June 1971, p. 1039.

3. G. R. Lambertson, D. Keefe, L. J. Laslett, W. A. Perkins, J. M. Peterson, and J. B. Rechen, ibid, p. 501.
4. A. G. Ruggiero, V. G. Vaccaro, Solution of the Dispersion Relation for Longitudinal Stability of an Intense Coasting Beam in a Circular Accelerator, CERN Report ISR-TH/68-33, 1968.

5. V. K. Neil and A. M. Sessler, Rev. Sci. Instr. 36, 429 (1965).

6. R. J. Briggs and V. K. Neil, Plasma Physics 8, 255 (1966).

7. A. Sessler and V. Vaccaro, Longitudinal Instabilities of Azimuthally Uniform Beams in Circular Vacuum Chambers with Walls of Arbitrary Electrical Properties, CERN Report 67-2, February 1967.

8. C. Pellegrini and A. M. Sessler, Nuovo Cimento X 53B, 198 (1968).

9. M. Abraham and R. Becker, The Classical Theory of Electricity and Magnetism, Blackie and Son, Limited, London and Glasgow (1968), p. 21l; S. Ramo, J. Whinnery, and T. van Duzer, Fields and Waves in Communication and Electronics, John Wiley and Sons, Inc., N. Y. (1967); or other convenient text.

10. V. K. Neil, Lawrence Livermore Laboratory, private communication. 